\title{
Can We Use MDDScore for the Identification of Comorbid Major Depressive Disorder (MDD) in Patients with Chronic Pain?
}

John $\mathrm{AB}^{1 *}$, Linda $\mathrm{MT}^{1}$, Joseph Shurman ${ }^{2}$ and Forest $\mathrm{ST}^{3}$

${ }^{1}$ Ridge Diagnostic Laboratories, Research Triangle Park, USA

${ }^{2}$ Scripps Memorial Hospital, Pain Management, 9888 Genesee Avenue La Jolla, CA, USA

${ }^{3}$ Veract Intractable Pain Clinic, 336 S Glendora Ave, West Covina, CA, USA

\begin{abstract}
The prevalence of patients with chronic pain who display depressive symptoms is quite high, with depression potentially an integral component of chronic pain. Since the current subjective clinical diagnostic systems were not designed to assess depression in patients with chronic pain, they fail to adequately capture the nature of mood states of these patients. It is difficult to apply these assessment tools to segregate unipolar depression (MDD) from the demoralization inherent in chronic pain states. MDDScore ${ }^{\mathrm{TM}}$, a multivariate biomarker blood test for depression, was used to determine if it was possible to identify biomarker patterns consistent with major depressive disorder in multiple chronic pain states. Three study groups were analyzed, and included: (i) patients $(n=93)$ with centralized Chronic Intractable Pain (CIP), (ii) patients $(n=20)$ with chronic pain of diverse origin from Scripps Pain Clinic (SPC) and (iii) prospectively collected patients $(n=28)$ with comorbid arthritis and depressive symptoms. A very distinct bimodal pattern was observed. Forty-nine of $93 \mathrm{CIP}$ patients $(52.7 \%), 18$ of $28(64.2 \%)$ of the arthritis patients, and 9 of $20(45 \%)$ patients with chronic pain of diverse origin from Scripps Pain Clinic had MDDScores of $\geq 5$. Thus, the biomarker panel could segregate patients into two major groups based upon MDDScores. These data suggest but not prove we can objectively identify chronic pain patients with a higher probability of comorbid major depression.

Importantly, we can use the biomarkers on the MDDScore panel to gain insight into and gauge the residual (post-treatment) level of inflammation in these intensively treated patients. To this end, we determined and compared the serum concentrations of alpha-1 antitrypsin (A1AT), Myeloperoxidase (MPO) and soluble tumor necrosis factor receptor type 2 (sTNFR2) in each of the patient populations studied.
\end{abstract}

Keywords: Clinical diagnostic; Demoralization; Biomarker blood test; Depression

\section{Introduction}

Approximately 100 million people in the United States suffer from chronic pain, which has been shown to adversely affect social relationships, work productivity, and quality of life, and is the leading cause of disability in the US [1-3]. The prevalence of patients with chronic pain who display depressive symptoms is quite high, with depression potentially an integral component of centralized pain [4-6]. Patients with chronic pain and depression have worse health outcomes than those with chronic pain alone [7-9]. One explanation for this finding is that psychological comorbidities, such as depression, can interfere with the effectiveness of pain treatment [10]. Prevalence studies have found that $18 \%$ to $56 \%$ of people with chronic pain suffer from a depressive disorder [3,5,9]. Pain patients often suffer from fatigue, anxiety, and poor quality of life; as such, they are frequently somatically focused $[11,12]$. Therefore, while the validity of self-reporting depression scales has been established in psychiatric settings, it is not clear that they are adequate tools for chronic pain patients

Often antidepressants, while not specifically intended to treat chronic pain, are used in the treatment of many chronic pain conditions, even when depression isn't recognized as a factor. One reason for that lack of recognition of comorbid depression is that depression scales and/or clinical psychological evaluations are not routinely employed in chronic pain patient care. A convenient and accurate quantitative test to serve as a depression diagnostic tool in chronic pain patients is needed. To this end, Bilello et al. described a biomarker panel (MDDScore; San Diego, CA) which can be used as an aid in MDD diagnosis [13]. It consists of nine biomarkers associated with the neurotrophic, metabolic, inflammatory, and hypothalamic-pituitary-adrenal (HPA) axis pathways. The neurotrophic pathway is represented by one biomarker (Brain Derived Neurotrophic factor; BDNF) while the metabolic pathway is represented by three biomarkers (prolactin $\{\mathrm{PRL}\}$, resistin $\{$ RETN\}, Apolipoprotein $\mathrm{C} 3\{\mathrm{APOC} 3\}$ ). Biomarkers for the inflammatory pathway (alpha-1 antitrypsin $\{\mathrm{A} 1 \mathrm{AT}\}$, myeloperoxidase $\{\mathrm{MPO}\}$ and soluble Tumor Necrosis Factor alpha receptor type II \{sTNFR2\}) are more frequently associated with chronic inflammation as opposed to acute inflammation. The HPA axis pathway is represented by two biomarkers, epidermal growth factor (EGF) and cortisol. This panel, plus its associated proprietary algorithm, has produced good sensitivity and specificity in differentiating MDD patients from normal subjects.

The first objective of this study was to determine if we can use the MDDScore test to identify pain patients with a physiological profile consistent with major depressive disorder and discriminate them from those who are demoralized by pain and display a depressed mood. Secondly, we were interested in assessing the serum levels of chronic inflammatory biomarkers within the MDDScore panel in patients with chronic pain in order to gain insight into and gauge the residual (posttreatment) level of inflammation in chronic pain states.

*Corresponding author: John AB, Ridge Diagnostic Laboratories, Research Triangle Park, USA, Tel: 440-465-3392; E-mail: jbilello@ridgedxlabs.com

Received December 09, 2016; Accepted December 26, 2016; Published December 28, 2016

Citation: John AB, Linda MT, Shurman J, Forest ST (2017) Can We Use MDDScore for the Identification of Comorbid Major Depressive Disorder (MDD) in Patients with Chronic Pain? J Mol Biomark Diagn 8: 319. doi: 10.4172/2155-9929.1000319

Copyright: ( 2017 John AB, et al. This is an open-access article distributed under the terms of the Creative Commons Attribution License, which permits unrestricted use, distribution, and reproduction in any medium, provided the original author and source are credited. 


\section{Methods}

\section{Pain patient population}

Extrahepatic The three study groups analyzed included: (a) patients with centralized intractable pain (CIP) from the Veract Intractable Pain Clinic $(\mathrm{n}=93)$, (b) patients with chronic pain of from the Scripps pain clinic (SPC) $(n=20)$, and $(c)$ a series of prospectively collected patients with comorbid arthritis and depressive symptoms $(n=28)$ were from multiple sources. Samples were collected from patients during the years 2012-2014 and were stored at a temperature lower than $-80^{\circ}$ prior to assay.

The gender and age distribution of the subjects are found in Table 1 below.

The arthritis group was a prospective collection of patients who presented with depressed mood and whose clinicians listed arthritis as a comorbidity upon submission for testing at Ridge Diagnostics. Nineteen patients $(68 \%)$ were female and nine $(32 \%)$ were male. The average BMI for females was $31 \pm 5.9$ (range 20.2-45.5). Males had an average BMI of $27.3 \pm 5.9$ (range 18.4-35.5).

The CIP study group consisted of patients diagnosed with centralized intractable pain by Dr. Tennant at the Veract pain clinic. Fifty-nine (63\%) were female and $34(37 \%)$ were male. The average BMI for females was $26.5 \pm 7.8$ (range from 13.3-48.7). Males had an average BMI of $27.9 \pm 4.8$ (range 19.6-40.9).

The SPC patients represent a diverse group of Dr. Shurman's patients. They included those diagnosed with post-laminectomy syndrome $(n=5)$, fibromyalgia $(n=4)$, degenerative disc disease $(n=3)$, neuralgic headache $(n=2)$ and other chronic pain states $(n=6)$. Eleven patients (55\%) were female and $9(45 \%)$ were male. The average BMI for females was $28.5 \pm 6.9$ (range 20.9-43.3). Males had an average BMI of $27.3 \pm 2.8$ (range 22.3-28.1).

Patients 18 years and younger were excluded. However, there was no specific exclusion criteria for pain patients both genders and all comorbidities were permitted. All medications were allowed, while they represent potential confounders, they were substantially diverse and more often than not used in combination and essential for the patient's wellbeing. Non-depressed subjects were excluded if they had previously been diagnosed with depression or were taking antidepressants. MDD patients were diagnosed with major depression as previously reported [13].

\section{Sample collection and handling}

Each study subject provided a blood sample, which was processed to collect serum. The sites prepared serum under standardized conditions. Briefly, blood was allowed to clot for 30 minutes, centrifuged 10 minutes at $1300 \times \mathrm{g}$ (RCF: relative centrifugal force) to collect serum that was aliquoted within 30 minutes of centrifugation. Serum samples were promptly shipped at $4^{\circ} \mathrm{C}$ (or frozen at $-80^{\circ} \mathrm{C}$ until ready for shipment on dry ice) to the Ridge Diagnostics CLIA Laboratory (Research Triangle Park, NC). The date and time of the blood draw was recorded by the study site for each sample, along with subject gender, height, and weight. All samples were identified by a sequentially applied accession number which blinded the technician doing testing to the source of the sample and patient identifiers.

\begin{tabular}{|c|c|c|c|c|}
\hline Group & Females & Males & Age & Range \\
\hline Arthritis & 19 & 9 & $70.2 \pm 11$ & $46-89$ \\
\hline CIP & 59 & 34 & $47.4 \pm 11.3$ & $24-72$ \\
\hline SPC & 11 & 9 & $50.8 \pm 14.1$ & $23-76$ \\
\hline \multicolumn{5}{r}{ Table 1: Patient demographics. }
\end{tabular}

\section{Serum biomarker assays}

Serum levels of the 9 biomarkers in the MDDScore panel, (A1AT, ApoC3, BDNF, Cortisol, EGF, MPO, PRL, RETN, and sTNFR2), were measured by validated individual quantitative immunoassays. Standard curves for calibrating the quantity of each biomarker were generated by non-serial dilution of each purified protein. Normal reference ranges (mean $\pm 2 \mathrm{SD}$ ) for each biomarker were previously determined from a large healthy control group; gender specific differences in normal reference ranges were observed for some analytes. Both A1AT and ApoC3 concentrations were measured by an analytically validated immunoturbidimetric assay developed at the Ridge Laboratory. BDNF and sTNFR2 were quantified using ELISA kits (Quantikine, R\&D Systems/BioTechne, Minneapolis, MN). EGF and RETN were quantified by an ELISA developed at the Ridge Laboratory (reagents from R\&D Systems/BioTechne, Minneapolis, MN). MPO was quantified using an ELISA kit (ALPCO, Salem, NH). PRL was quantified using a human serum ELISA, and Cortisol was quantified using a competition ELISA (Monobind, Lake Forest, CA).

The sensitivity and specificity of the MDDScore panel and algorithm are $94 \%$ and $92 \%$ and the area under the ROC curve (AUC) is 0.96 [13]

\section{Cortisol adjustments for diurnal variation}

It is well known that serum cortisol levels undergo a diurnal variation with peak levels occurring between 6-8 A.M. that gradually fall over the course of the day, reaching a lowest point around midnight. While the majority of subjects had their blood drawn for MDDScore testing in the late morning or early afternoon, when the decline in cortisol was linear, patients drawn early in the day (particularly 30-45 minutes after awakening) had peak levels. To control for this variation, we developed a method to convert all cortisol values to a noon-time equivalent (CORTcorr) using a concentration time curve derived from studies of diurnal variation in normal subjects and MDD patients [14,15].

\section{Calculations}

Age, gender, height, and weight of each subject was recorded and BMI was calculated using the formula $\mathrm{BMI}=($ weight in lbs. $\times 703) /$ (height in inches)2 and an online calculator (http://www.mayoclinic. com/health/bmi-calculator/NU00597). The MDDScore algorithm was as previously described [13].

The full algorithmic model is: $\mathrm{Dx} \sim$ Gender+CORTcorr $+\mathrm{BDNF}+1$ og $(\mathrm{MPO})+\log (\mathrm{EGF})+$ Gender $^{*}(\mathrm{PRL}+\mathrm{RETN}+\mathrm{sTNFR} 2+\mathrm{BMI}+$ Age $)+\mathrm{A} 1$ $\mathrm{AT}+\mathrm{APOC}$

Where, Gender ${ }^{*}($ PRL + RETN + sTNFR $2+$ BMI + Age $)$ directs the model to include an interaction term between Gender and each of the variables PRL, RETN, sTNFR2, BMI and age as well as those variables themselves. Dx is the prediction of the diagnosis (MDD or not-MDD) of the subject that we obtain using the independent variables to the right of the $\sim$. The use of $\sim$ to specify the model as opposed to $=$ is that for logistic regression used here, the independent variables don't directly predict/estimate Diagnosis (MDD vs. non-MDD) the dependent variable. Instead they estimate some other quantity (log-odds of having MDD, or the MDDScore, in our case) that predicts the diagnosis.

$\mathrm{P}$ values were calculated in excel using the TTEST function and assuming two tails and two samples of equal variance (homoscedastic).

\section{Results}

\section{MDDScores in patients with chronic pain}

The MDDScore utilizes the resultant serum concentrations of all of 
the biomarkers on the MDDScore panel in an algorithm which provides a single score between 1 and 9 [16]. Figure 1 shows the distribution of MDDScores in each of the three patient populations. The distribution of scores was not Gaussian. A very distinct bi-modal pattern was observed. Overall 43.5\% scored 1-3, 8\% scored 3-6 and 48.5\% scored 7-9. Eighteen of 28 patients with comorbid arthritis (64.2\%) had MDDScores greater or equal to 5 . Forty-nine of 93 CIP patients (52.7\%), 18 of $28(64.2 \%)$ of the arthritis patients, and 9 of $20(45 \%)$ patients with chronic pain of diverse origin from Scripps Pain Clinic had scores of $\geq 5$. This is in contrast to an earlier prospective study using the same algorithm and assay technology, wherein $93.7 \%$ of well characterized MDD patients had an MDDScore greater or equal to 5; $91.8 \%$ of control patients had scores of $<5$ [13] (Figure 1).

The MDDScore panel of 9 biomarkers and algorithm were used to determine the MDDScore in each group of pain patients. The test scores ranged from 1 to 9. An MDDScore of 1 to 4 suggests a low probability or likelihood of the patient having depression. An MDDScore of 5 to 9 suggests the patient has a high probability or likelihood of MDD [13].

The histogram above shows the distribution of MDDScores in patients with chronic pain. The majority of the patients had and MDDScores of 1 or 9 , indicative that patients with chronic pain exhibited a bimodal pattern.

Since the MDDScore algorithm incorporates gender and BMI as clinical parameters, we also looked at the distribution of MDDScores by gender and BMI. Table 2 shows the distribution of MDDScores $<5$ and $\geq 5$ in the pain populations, by gender. Table 2 shows the percentage of male and female pain patients with MDDScores of $<5$ or $\geq 5$. While

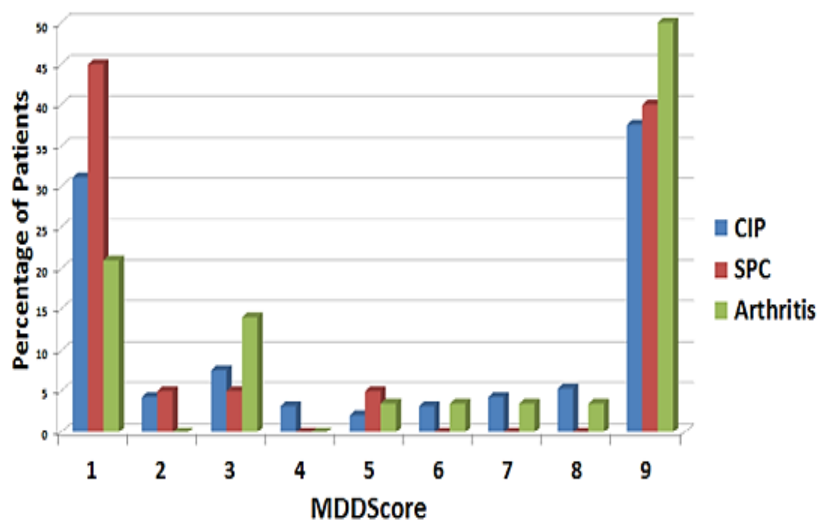

Figure 1: Distribution of MDD scores in chronic pain populations.

\begin{tabular}{|c|c|c|c|c|}
\hline & \multicolumn{2}{|c|}{ Males (\%) } & \multicolumn{2}{c|}{ Females (\%) } \\
\hline Group & $<\mathbf{5}$ & $\mathbf{2 5}$ & $<\mathbf{5}$ & $\mathbf{2 5}$ \\
\hline Arthritis & 31.6 & 68.4 & 44.4 & 55.6 \\
\hline CIP & 54.5 & 44.5 & 42.4 & 57.6 \\
\hline SPC & 44.4 & 55.6 & 63.6 & 37.4 \\
\hline
\end{tabular}

Table 2: Distribution of MDD scores by gender.

\begin{tabular}{|c|c|c|c|c|}
\hline Group & $\mathbf{n}$ & Mean & Std Dev & Range \\
\hline Arthritis & 28 & 138.7 & 28.8 & $74-201$ \\
\hline CIP & 93 & 183 & 38.9 & $105-281$ \\
\hline SPC & 20 & 130.2 & 42.1 & $71-215$ \\
\hline Non-Depressed & 86 & 148.9 & 35.2 & $55-259$ \\
\hline MDD Patients & 68 & 156.0 & 37.3 & $92-257$ \\
\hline
\end{tabular}

Table 3: Serum concentrations of alpha-1 antitrypsin ( $\mathrm{mg} / \mathrm{dL})$.

\begin{tabular}{|c|c|c|c|c|}
\hline Group & $\mathbf{n}$ & Mean & Std Dev & Range \\
\hline Arthritis & 28 & 192.5 & 101.3 & $88-539$ \\
\hline CI P & 93 & 352.4 & 164.9 & $105-928$ \\
\hline SPC & 20 & 238.3 & 119.0 & $102-488$ \\
\hline Non-Depressed & 86 & 188.2 & 107.9 & $80-567$ \\
\hline MDD Patients & 68 & 273.5 & 178.7 & $89-925$ \\
\hline
\end{tabular}

Table 4: Serum concentration of myeloperoxidase $(\mathrm{ng} / \mathrm{mL})$.

\begin{tabular}{|c|c|c|c|c|}
\hline Group & $\mathbf{n}$ & Mean & Std Dev & Range \\
\hline Arthritis & 28 & 4861.5 & 2537.7 & $2167-13654$ \\
\hline CIP & 93 & 3078.9 & 1109.4 & $1154-7727$ \\
\hline SPC & 20 & 2559.2 & 947.2 & $1479-4614$ \\
\hline Non-Depressed & 86 & 2548.1 & 666.7 & $1633-5798$ \\
\hline MDD Patients & 68 & 2550.7 & 681.9 & $1428-4810$ \\
\hline
\end{tabular}

Table 5: Serum concentrations of soluble tumor necrosis factor receptor type 2 (pg/mL).

\begin{tabular}{|c|c|c|c|c|c|}
\hline & Arthritis & SPC & CIP & Normal & MDD \\
\hline Arthritis & - & 0.39 & $<0.0001$ & 0.16 & 0.03 \\
\hline SPC & 0.39 & - & $<0.0001$ & 0.036 & 0.01 \\
\hline CIP & $<0.0001$ & 0.05 & - & 0.0001 & $<0.0001$ \\
\hline Normal & $<0.0001$ & 0.036 & $<0.0001$ & - & 0.23 \\
\hline MDD & $<0.0001$ & 0.009 & $<0.0001$ & 0.23 & - \\
\hline
\end{tabular}

Table 6: $P$ values for serum concentrations of alpha- 1 antitrypsin between study groups.

\begin{tabular}{|c|c|c|c|c|c|}
\hline & Arthritis & SPC & CIP & Normal & MDD \\
\hline Arthritis & - & 0.158 & $<0.0001$ & 0.854 & 0.025 \\
\hline SPC & 0.158 & - & 0.004 & 0.069 & 0.409 \\
\hline CIP & $<0.0001$ & 0.004 & - & $<0.0001$ & 0.004 \\
\hline Normal & 0.854 & 0.069 & $<0.0001$ & - & 0.004 \\
\hline MDD & $<0.0001$ & 0.009 & 0.004 & 0.0003 & - \\
\hline
\end{tabular}

Table 7: $P$ values for serum concentrations of myeloperoxidase between study groups.

there are differences in the absolute distribution, there is no significant bias of MDDScore in male and female patients.

No significant differences in BMI were seen between female patients with MDDScores $<5(26.6 \pm 7.2)$ and those with $\geq 5(28.6 \pm$ 7.7), $\mathrm{p}=0.21$. Similarly, there were no significant differences in BMI in male pain patients with MDDScores $<5(27.1 \pm 4.4)$ and those with scores $\geq 5(27.8 \pm 4.4), \mathrm{p}=0.53$.

\section{Chronic inflammatory proteins in patients with chronic pain}

The MDDScore test panel measures the serum concentrations of three biomarkers of chronic inflammation: A1AT, MPO, and sTNFR2. Tables 3-5 show the mean serum concentrations of each chronic inflammatory biomarker for the pain patients. The chronic pain patient data is presented along with that of MDD patients and non-depressed controls from our earlier study [13].

It is clear from Tables 3-5, that there are differences in the serum concentration of A1AT, MPO, and sTNFR2 between groups of subjects. The CIP patients had the highest serum levels of A1AT and MPO, while patients with arthritis had the highest concentrations of circulating sTNFR2. Probability ( $p$ values) were calculated using the Student TTEST to assess the statistical significance of differences in serum concentration of each biomarker between the chronic pain populations, healthy non-depressed subjects, and well-characterized MDD patients. Tables 6-8 indicate the $\mathrm{p}$ values for A1AT, MPO, and sTNFR2 between the chronic pain groups and the normal subjects and MDD patients 


\begin{tabular}{|c|c|c|c|c|c|}
\hline & Arthritis & SPC & CIP & Normal & MDD \\
\hline Arthritis & - & 0.0005 & $<0.0001$ & $<0.0001$ & $<0.0001$ \\
\hline SPC & 0.0005 & - & 0.05 & 0.95 & 0.96 \\
\hline CIP & $<0.0001$ & 0.05 & - & 0.0001 & 0.0005 \\
\hline Normal & $<0.0001$ & 0.95 & 0.0001 & - & 0.98 \\
\hline MDD & $<0.0001$ & 0.96 & 0.0005 & 0.98 & - \\
\hline
\end{tabular}

Table 8: $P$ values for serum concentrations of soluble TNF receptor type 2 between study groups.

from our earlier study [13]. CIP patients were significantly different from all other groups of patients.

\section{Discussion}

It has been shown that psychiatric illness, particularly unipolar depression or MDD, is actually a common comorbidity $(30 \%$ to $60 \%$ ) in chronic pain patients, often confounding a full diagnosis of those individuals. MDDScore is a multivariate biomarker assay plus a proprietary algorithm that provides a score based upon the serum levels of biomarkers of the inflammatory, HPA axis, metabolic and neurotrophic pathways. Our results indicate that MDDScore was able to segregate seemingly diverse populations of pain patients into two groups. From $45 \%$ to $65 \%$ of the pain patients had MDDScores $\geq 5$ indicative of depression. This suggests that MDDScore may be able to identify patients with a higher probability of comorbid major depression. These data are in contrast to well-characterized MDD patients where 65 of 68 patients $(95.5 \%)$ had MDDScores $\geq 5$ [13]. In that same study, 9 of 86 (8.1\%) non-depressed subjects had MDDScores $\geq 5$. This comparison suggests that approximately half of the chronic pain patients had a serum biomarker profile consistent with patients with MDD that we had previously characterized. It is important to note that in our survey of three diverse patient populations with chronic pain, $45 \%$ to $65 \%$ had high MDDScores which would be consistent with longstanding estimates (30\% to $60 \%$ ) of comorbid depression in chronic pain patients $[3,5,9]$. While this study suggests that MDDScore could be useful in objectively identifying chronic pain patients with depression, it does not a priori establish that clinical utility. Other studies in pain patients with well-established MDD diagnoses appear to be required. Studies of the response of patients with high and low MDDScores to antidepressant treatment may be useful in this regard since it is known that treatment decreases in pain will be associated with antidepressant depression response [17].

While not specifically developed to treat chronic pain, certain antidepressants such as those with serotonin or norepinephrine reuptake inhibition (SSRI, SNRIs) play an important role in the treatment of many chronic pain conditions, even when comorbid depression isn't recognized [18-21]. Indeed, tricyclic antidepressants are considered first-line systemic therapy for many neuropathic pain syndromes. Most of the chronic pain patients in this study have or have had antidepressants as part of their therapeutic regimen. However, the incorporation of an antidepressant into the patient's treatment regimen was often an empirical decision. Nonetheless, the extent to which depression is due to psychological factors and to what extent is it due to a biochemical disturbance is an extremely important distinction in guiding treatment decisions. In this regards Preston and Johnson in "Clinical Psychopharmacology" made the following comment: "Most purely psychological problems are not helped by medication treatment. On the other hand, most biologically based psychiatric disorders require medication treatment" [22].

In this study of three pain populations we also gained physiological information on biomarker expression patterns in chronic pain states.
The largest group of patients we evaluated were those with central syndromes wherein sensitization of the pain system occurs that can either be relegated to a specific part of the body or encompass the entire body. Central pain is typically constant, and may be moderate to severe in intensity. CIP patients, as a group, have been shown to have a more severe, persistent pain that usually fail to respond to non-narcotic analgesics and other treatment measures [23,24]. We also examined expression in the Scripps Pain Clinic group of patients who had both localized and diffuse pain symptomatology including post-laminectomy syndrome, neuralgic headache, fibromyalgia, and degenerative disc disease. In some respect the SPC group is one where pain was more localized. Lastly, we had access to patients with depressed mood and comorbid arthritis which suggested that their pain was primarily localized to joints such as the back (most common), knee or neck. We used the MDDScore biomarker panel to measure and compare the expression of three chronic inflammatory biomarkers in each group of chronic pain patients; and in comparison to previously determined values for MDD patients and normal subjects. Alpha-1 antitrypsin (A1AT), an acute phase protease inhibitor belonging to the serpin superfamily, protects tissues from elastase and other enzymes released from inflammatory cells [25]. Myeloperoxidase (MPO) is an inflammatory peroxidase enzyme most abundantly present in neutrophil granulocytes [26,27]. Lastly, we measured serum levels of sTNFR2, a circulating form of the receptor for TNF alpha involved in reducing systemic inflammation by binding TNF [28].

While there are some clinical tests for inflammation e.g. measurement of C-reactive protein or erythrocyte sedimentation rate (ESR) they are generally non-specific: particularly with regards to chronic inflammation $[29,30]$. Tables $3-5$ show the mean serum concentration of each of the inflammatory biomarkers. The highest mean serum concentration of A1AT and MPO were seen in CIP patients, while the highest mean serum level of sTNFR2 was observed in patients with arthritis. All of the patients with chronic pain we studied were being treated, often with a variety of medications both non-narcotic and narcotic. Our observation that subsets of the three inflammatory biomarkers can be expressed in these aggressively treated patients suggests that despite treatment residual inflammation is present. It should be pointed out that A1AT and sTNFR2 are anti-inflammatory mediators which circulate systemically at reasonable concentration to block pro-inflammatory molecules released by inflammatory cells $[25,28]$. In contrast, MPO is a well-known enzyme, mainly released by activated neutrophils, characterized by powerful pro-oxidative and proinflammatory properties $[25,26]$. MPO's role in both psychiatry and pain may be related to its elaboration from glial cells [31-34]. To that end patients with arthritis, a localized response, had the lowest mean levels of serum MPO and the highest mean levels of sTNFR2. Statistical analysis of the biomarker data (Tables 6-8) indicated that the CIP patients as a group were significantly different from SPC and arthritis patients in the serum levels of all three biomarkers. Levels of A1AT, MPO, and sTNFR2 in the serum of CIP patients were also significantly different from MDD patients and normal subjects. With the exception of sTNFR2 serum levels, arthritis patients were not significantly different from the patients from SPC. Clearly the size of the SPC and arthritis groups were smaller than we would have liked, and while we identified residual inflammation in each group, larger groups of well characterized patients would need to be evaluated to see if differences in biomarker expression between groups can be established. It should be pointed out that we have focused upon mean serum biomarker concentrations, and as noted in Tables 3-5. There is a range of concentrations in each patient group such that it may be difficult to segregate CIP patients with 
Citation: John AB, Linda MT, Shurman J, Forest ST (2017) Can We Use MDDScore for the Identification of Comorbid Major Depressive Disorder (MDD) in Patients with Chronic Pain? J Mol Biomark Diagn 8: 319. doi: 10.4172/2155-9929.1000319

Page 5 of 5

centralized pain from other patients with other types of chronic pain. Nonetheless, use of the MDDScore test which includes biomarkers of chronic inflammatory processes may provide better clinical evidence for continued inflammation and tissue damage. Knowledge of residual inflammation may provide guidance to clinicians treating patients with chronic pain and may aid in designing regimens targeting persistent inflammation.

Future studies will tell us whether an objective, physiological tool such as MDDScore would be clinically useful in the identification of patients with physiologically based depression who may benefit from having an antidepressant added to their therapeutic regimen, versus those patients whose depressed mood is one better characterized as an Adjustment Disorder and will likely benefit more from counseling.

\section{Acknowledgement}

We would like to acknowledge the support of Ridge Diagnostics who provided partial funding for this study. In addition, we thank Bob Paradowski and Lori Henderson, former colleagues at Ridge Diagnostics, who provided valuable discussions and critique. We acknowledge the excellent laboratory assistance of Ms. Laurie Rotchford MLS who performed most of the assays reported in this study.

\section{References}

1. American Chronic Pain Association. Pain fact sheet. 2007.

2. Institute of Medicine Report from the Committee on Advancing Pain Research, Care, and Education: Relieving pain in America, a blueprint for transforming prevention, care, education and research. The National Academies Press; 2011.

3. Tsang A, Von Korff M, Lee S, Alonso J, Karam E, et al. (2008) Common chronic pain conditions in developed and developing countries: Gender and age differences and comorbidity with depression-anxiety disorders. J Pain $9(10)$ : 883-891.

4. Korff MV, Simon G (1996) The relationship between pain and depression. Br J Psychiatry 168(30): 101-108.

5. Bair MJ, Robinson RL, Katon W, Kroenke K (2003) Depression and pain comorbidity: A literature review. Arch Intern Med 163(20): 2433-2445.

6. Nekovarova T, Yamamotova A, Vales K, Stuchlik A, Fricova J, et al. (2014) Common mechanisms of pain and depression: Are antidepressants also analgesics? Front Behav Neurosci 8: 1-12.

7. Moussavi S, Chatterji S, Verdes E, Tandon A, Patel V, et al. (2007) Depression, chronic diseases, and decrements in health: results from the World Health Surveys. Lancet 370: 851-858.

8. Bair MJ, Wu J, Damush TM, Sutherland JM, Kroenke K (2008) Association of depression and anxiety alone and in combination with chronic musculoskeletal pain in primary care patients. Psychosom Med 70(8): 890-897.

9. Arnow BA, Hunkeler EM, Blasey CM, Lee J, Constantino MJ, et al. (2006) Comorbid depression, chronic pain, and disability in primary care. Psychosom Med 68: 262-268.

10. Lindsay PG, Wyckoff M (1981) The depression-pain syndrome and its response to antidepressants. Psychosomatics 22: 571-573.

11. Von Korff M, Dworkin SF, Le Resche L, Kruger A (1988) An epidemiologic comparison of pain complaints. Pain 32: 173-183.

12. Morley S, Williams ACDC, Black S (2002) A confirmatory factor analysis of the Beck depression inventory in chronic pain. Pain 99: 289-298.

13. Bilello JA, Thurmond LM, Smith KM, Pi B, Rubin R, et al. (2015) MDDScore Confirmation of a blood test to aid in the diagnosis of major depressive disorder. J Clin Psychiatry 76: e199-206.

14. Cizza G, Ronsaville DS, Kleitz H, Eskandari F, Mistry S, et al. (2012) Clinica subtypes of depression are associated with specific metabolic parameters and circadian endocrine profiles in women: The power study. PloS one 7: e28912.

15. Young EA, Carlson NE, Brown MB (2001) Twenty-four-hour ACTH and cortisol pulsatility in depressed women. Neuro psycho pharmacology 25: 267-76.

16. Smith KM, Renshaw PF, Bilello J (2013) The diagnosis of depression: Current and emerging methods. Compr Psychiatry 54(1): 1-6.

17. Fishbain DA, Cole B, Lewis JE, Gao J (2014) Does pain interfere with antidepressant depression treatment response and remission in patients with depression and pain? An evidence-based structured review. Pain Med 15 1522-1539.

18. McQuay HJ, Tramer M, Nye BA, Carroll D, Wiffen PJ, et al. (1996) A systematic review of antidepressants in neuropathic pain. Pain 68: 217-227.

19. Ansari A (2000) The efficacy of newer antidepressants in the treatment of chronic pain: A review of current literature. Harvard review of psychiatry 7: 257-277.

20. Turk DC, Wilson HD, Cahana A (2011) Treatment of chronic non-cancer pain. Lancet 377: 2226-2235.

21. Gilron I, Jensen TS, Dickenson AH (2013) Combination pharmacotherapy for management of chronic pain: from bench to bedside. Lancet Neurol 12: 1084-1095.

22. Preston J, Johnson J (2011) Clinical psychopharmacology. Medmaster Inc.

23. Tennant F, Hermann L (2000) Intractable or chronic pain: There is a difference. West J Med 173: 306.

24. Tennant FS, Robinson D, Sagherian A, Seecof R (1988) Chronic opioid treatment of intractable non-malignant pain. NIDA Res Monogr 81: 174-180.

25. Gettins PG (2002) Serpin structure, mechanism, and function. Chem Rev 102 4751-4804.

26. Arnhold J (2004) Properties, functions, and secretion of human myeloperoxidase. Biochemistry (Moscow) 69: 4-9.

27. van der Veen BS, de Winther MP, Heeringa P (2009) Myeloperoxidase: Molecular mechanisms of action and their relevance to human health and disease. Antioxid Redox Signal 11: 2899-2937.

28. Van Zee KJ, Kohno T, Fischer E, Rock CS, Moldawer LL, et al. (1992) Tumo necrosis factor soluble receptors circulate during experimental and clinical inflammation and can protect against excessive tumor necrosis factor alpha in vitro and in vivo. Proc Natl Acad Sci U S A 89: 4845-4849.

29. Tennant F, Hermann L (2002) Using biologic markers to identify legitimate chronic pain. Am Clin Lab 21: 14

30. Tennant $F$ (2013) Erythrocyte sedimentation rate and c-reactive protein: Old but useful biomarkers for pain treatment. Practical Pain Management.

31. Vaccarino V, Brennan ML, Miller AH, Bremner JD, Ritchie JC, et al. (2008) Association of major depressive disorder with serum myeloperoxidase and other markers of inflammation: A twin study. Biol Psychiatry 64: 476-483.

32. Dublin $P$, Hanani M (2007) Satellite glial cells in sensory ganglia: Their possible contribution to inflammatory pain. Brain Behav Immun 23: 592-598.

33. Gray E, Thomas TL, Betmouni S, Scolding N, Love S (2008) Elevated activity and microglial expression of myeloperoxidase in demyelinated cerebral cortex in multiple sclerosis. Brain Pathol 18: 86-95.

34. Choi DK, Pennathur S, Perier C, Tieu K, Teismann P, et al. (2005) Ablation of the inflammatory enzyme myeloperoxidase mitigates features of Parkinson's disease in mice. J Neurosci 25: 6594-6600. 